\title{
Endobronchial elastography in the evaluation of esophageal invasion
}

\author{
Terunaga Inage, MD, Takahiro Nakajima, MD, PhD, Shigetoshi Yoshida, MD, PhD, and \\ Ichiro Yoshino, MD, PhD
}

Video clip is available online.

Because a primary tracheal tumor occasionally invades surrounding structures, such as the thyroid, esophagus, and great vessels, pretreatment evaluation is important for surgical planning. The usual imaging modalities for tracheal tumors include computed tomography (CT), magnetic resonance imaging, and endoscopic ultrasound (EUS). ${ }^{1}$ However, the resolving power of these modalities can be unsatisfactory because of the anatomy of this area. Although EUS was reported to be useful for evaluating malignant invasion of the mediastinum, ${ }^{2}$ obtaining an image with a clear distinction between the border of a tumor and the surrounding tissue using EUS is difficult.

From the Department of General Thoracic Surgery, Graduate School of Medicine, Chiba University, Chiba, Japan.

This work was supported by a JFE (The Japanese Foundation for Research and Promotion of Endoscopy) Grant for preparing the submission. The ultrasound scanner (EU-Y0008) was approved for clinical use by the Ministry of Health, Labour and Welfare in Japan. We leased the EU-Y0008 from Olympus Medical Systems (Tokyo, Japan).

Disclosures: Takahiro Nakajima received honoraria and lecture fees from Olympus Medical Systems (Tokyo, Japan) for endobronchial ultrasound transbronchial needle aspiration training courses. All other authors have nothing to disclose with regard to commercial support.

Received for publication June 27, 2014; revisions received Oct 13, 2014; accepted for publication Oct 16, 2014; available ahead of print Nov 13, 2014.

Address for reprints: Takahiro Nakajima, MD, PhD, Department of General Thoracic Surgery, Graduate School of Medicine, Chiba University, 1-8-1 Inohana, chuo-ku, Chiba 260-8670, Japan (E-mail: takahiro_nakajima@med.miyazaki-u.ac.jp).

J Thorac Cardiovasc Surg 2015;149:576-7

$0022-5223 / \$ 36.00$

Copyright (C) 2015 by The American Association for Thoracic Surgery

http://dx.doi.org/10.1016/j.jtcvs.2014.10.080
Elastography is a strain imaging technique that assesses tissue stiffness and creates a visual representation of the distributions of stiffness in the region of interest. Tissue stiffness is estimated by measuring the strain of the tissue in response to mechanical stress, either local compression or vibratory. To measure the amount of change before and after an area is compressed, we can calculate the tissue stiffness. The feedback on tissue stiffness is converted into a colorcoded image that is displayed on the ultrasound image; areas of stiffness can, for example, appear in blue, and soft areas in red. Here, we report on a patient with a tracheal tumor and an extraluminal extension that were successfully assessed before surgery using endobronchial elastography. Elastography showed that the tumor had not invaded the esophagus.

\section{CLINICAL SUMMARY}

A 60-year-old woman suddenly developed difficulty breathing. Chest CT showed a large tracheal tumor, with the left main bronchus almost completely obstructed by the tumor (Figure 1,A). She subsequently developed obstructive pneumonia and severe respiratory failure. We performed transbronchial debulking of the residual tumor. Contrast chest CT revealed residual extraluminal wellcontrasted tumor that was adjacent to the esophagus (Figure 1, B). Positron emission tomography showed high levels of accumulation of ${ }^{18} \mathrm{~F}$ - fludeoxyglucose in the tumor and a maximum standard uptake value of 29.6. The histopathologic diagnosis of biopsied tissue was low-grade malignant stromal tumor. Esophageal endoscopy showed normal esophageal mucosa with external compression by the tumor.

The patient underwent convex-probe endobronchial ultrasonography using the EU-Y0008 ultrasound scanner
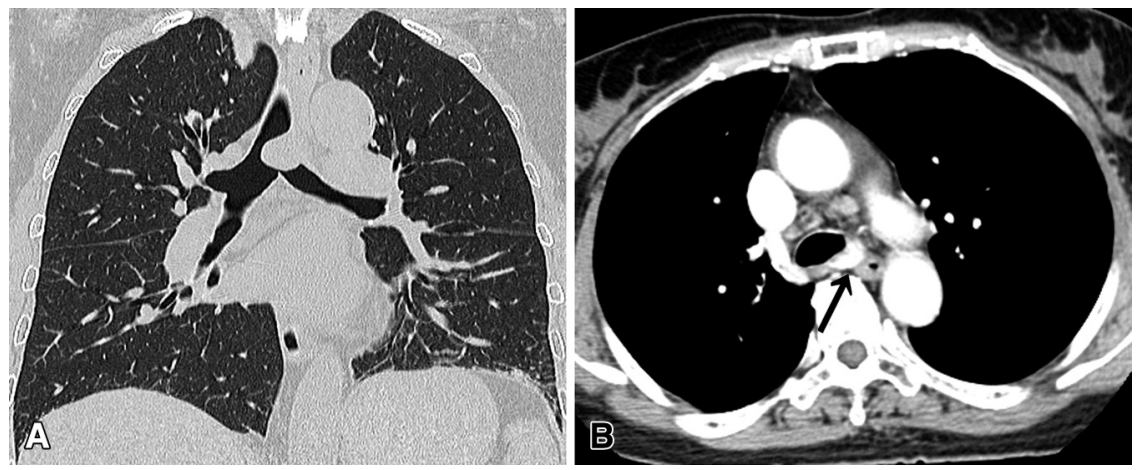

FIGURE 1. A, Chest CT shows an endotracheal mass with luminal narrowing and almost complete obstruction of the left main bronchus. B, Contrast chest CT reveals a well-contrasted residual tumor mainly in the extraluminal region of the trachea and adjacent to the esophagus (arrow). 

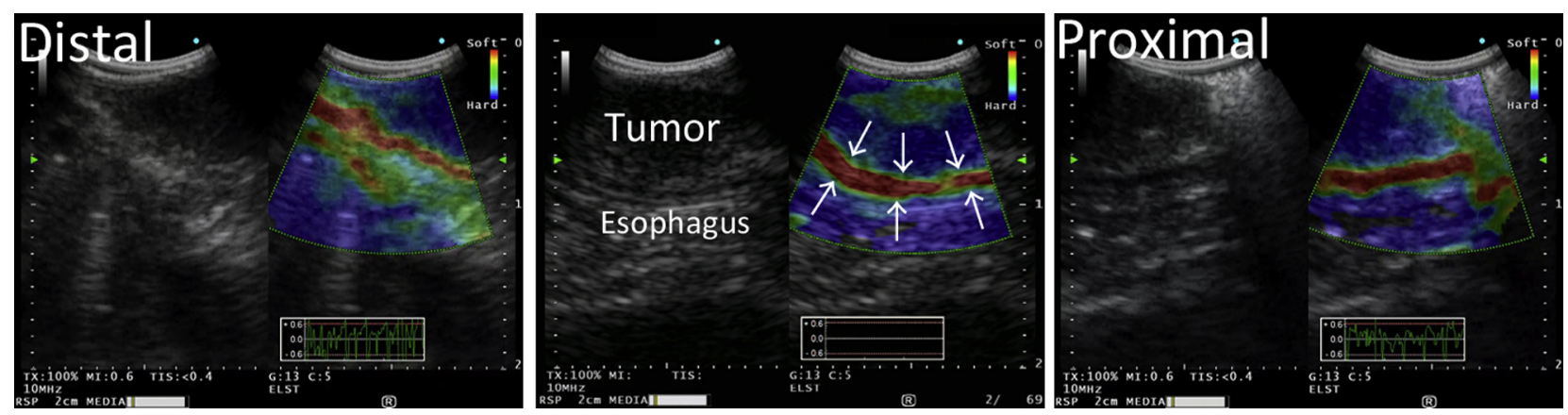

FIGURE 2. Elastography clearly shows the tumor and periesophageal fat tissue in different colors (the tumor is blue; the periesophageal fat tissue is red) (arrows). An intact border between the tumor and esophageal wall is clearly evident. Longitudinal transbronchial elastographic mapping from the distal margin to the proximal margin of the tumor showed preserved periesophageal fat tissue.

(Olympus Medical Systems, Tokyo) combined with the Olympus BF-UC260FW bronchofibervideoscope. Elastography clearly distinguished between the stiff tumor and periesophageal fat tissue, and the linear border between the 2 (Figure 2). Longitudinal transbronchial elastographic mapping from the distal margin to the proximal margin of the tumor showed preserved periesophageal fat tissue (Figure 2) and paradoxical movement between the tumor and esophagus (Video 1). These findings indicated that the tumor was localized and had not invaded the esophagus.

The patient underwent carinal resection and reconstruction using the double-barrel method. The tumor, along with the periesophageal fat tissue, was easily dissected from the esophagus. The final determination was that the resected tumor was an unclassified low-grade sarcoma.

\section{DISCUSSION}

In clinical practice, elastography provides visual information converted from the quantitative imaging of strain and the elastic modulus distributions. Elastic modulus is calculated by measuring the tendency of an object to deform along an axis when tissue is compressed. Malignant tissues tend to be stiffer than normal tissues because of the increased density of tumor cells and vascular structures, as well as fibrosis; therefore, this technology can be used to assess various organs for the presence of malignancy. ${ }^{3}$ This technology has been integrated with endoscopic devices. Endoscopic ultrasound elastography has been used to differentiate between benign and malignant lymph nodes in the mediastinum and abdominal cavity. ${ }^{4}$ Preliminary data on elastography integrated with convex-probe endobronchial ultrasonography was recently reported. ${ }^{5}$

The evaluation of tumors located in the respiratory tract, with regard to their invasion of surrounding organs, is generally important for surgical planning. Computed tomography and magnetic resonance imaging are commonly used for this purpose. However, ultrasound has been reported to be a high-priority modality for evaluating tumor invasion of the aorta. ${ }^{2}$ Guidelines from the American College of Chest Physicians indicate that EUS can be used to identify the direct invasion of the mediastinum by lung cancers. ${ }^{1}$ Ultrasound is a powerful tool for evaluating T4 tumors; however, the interpretation of B-mode imaging is often difficult. Elastography incorporated into an ultrasound system should be very useful for assessing whether a tumor has invaded the surrounding structures. The location of each structure is clearly shown in the colored image, and the border between tumor and surrounding tissue can be easily identified because of the color coding used to depict different degrees of tissue stiffness.

\section{CONCLUSIONS}

We treated a rare case of primary tracheal sarcoma with extraluminal extension. The border of the tumor in relation to the esophagus was evaluated using real-time elastography combined with convex-probe endobronchial ultrasound.

\section{References}

1. Silvestri GA, Gonzalez AV, Jantz MA, Margolis ML, Gould MK, Tanoue LT, et al Methods for staging non-small cell lung cancer: diagnosis and management of lung cancer, 3rd ed: American College of Chest Physicians evidence-based clinical practice guidelines. Chest. 2013;143:e211S-50S.

2. Varadarajulu S, Schmulewitz N, Wildi SM, Roberts S, Ravenel J, Reed CE, et al Accuracy of EUS in staging of T4 lung cancer. Gastrointest Endosc. 2004;59: 345-8.

3. Dietrich CF, Saftoiu A, Jenssen C. Real time elastography endoscopic ultrasound (RTE-EUS), a comprehensive review. Eur J Radiol. 2014;83:405-14.

4. Xu W, Shi J, Zeng X, Li X, Xie WF, Guo J, et al. EUS elastography for the differentiation of benign and malignant lymph nodes: a meta-analysis. Gastrointest Endosc. 2011;74:1001-9.

5. Trosini-Desert V, Jeny F, Taillade L, Vignot S, Zribi H, Capron F, et al. Bronchial endoscopic ultrasound elastography: preliminary feasibility data. Eur Respir J. 2013;41:477-9. 